\title{
Coronavirus Disease (COVID-19): The Value of Chest Radiography for Patients Greater Than Age 50 Years at an Earlier Timepoint of Symptoms Compared With Younger Patients
}

\author{
Mae Igi, MD, ${ }^{1}$ Molly Lieux, BS, ${ }^{2}$ Joe Park, MD, ${ }^{1}$ Catherine Batte, $M S,{ }^{3}$ Bradley Spieler, MD ${ }^{1}$ \\ ${ }^{1}$ Department of Diagnostic Radiology, Louisiana State University Health Sciences Center, New Orleans, LA ${ }^{2}$ Louisiana State University \\ Health Sciences Center, School of Medicine, New Orleans, LA ${ }^{3}$ Department of Physics and Astronomy, Louisiana State University, Baton \\ Rouge, LA
}

Background: A relative paucity of data exists regarding chest radiography (CXR) in diagnosis of coronavirus disease (COVID-19) compared to computed tomography. We address the use of a strict pattern of CXR findings for COVID-19 diagnosis, specifically during early onset of symptoms with respect to patient age.

Methods: We performed a retrospective study of patients under investigation for COVID-19 who presented to the emergency department during the COVID-19 outbreak of 2020 and had CXR within 1 week of symptoms. Only reverse transcription polymerase chain reaction (RT-PCR)-positive patients were included. Two board-certified radiologists, blinded to RT-PCR results, assessed 60 CXRs in consensus and assigned 1 of 3 patterns: characteristic, atypical, or negative. Atypical patterns were subdivided into more suspicious or less suspicious for COVID-19.

Results: Sixty patients were included: 30 patients aged 52 to 88 years and 30 patients aged 19 to 48 years. Ninety-three percent of the older group demonstrated an abnormal CXR and were more likely to have characteristic and atypical-more suspicious findings in the first week after symptom onset than the younger group. The relationship between age and CXR findings was statistically significant $\left(\chi^{2}[2, \mathrm{n}=60]=15.70 ; P=0.00039\right)$. The relationship between negative and characteristic COVID-19 CXR findings between the 2 age cohorts was statistically significant with Fisher exact test resulting in a $P$ value of 0.001 .

Conclusion: COVID-19 positive patients $>50$ years show earlier, characteristic patterns of statistically significant CXR changes than younger patients, suggesting that CXR is useful in the early diagnosis of infection. CXR can be useful in early diagnosis of COVID-19 in patients older than 50 years.

Keywords: Chest radiography, COVID-19, diagnostic imaging, pneumonia, x-rays

Address correspondence to Bradley Spieler, MD, Department of Diagnostic Radiology, Louisiana State University Health Sciences Center, 1542 Tulane Ave., Room 343, New Orleans, LA 70112. Tel: (504) 568-5523. Email: bspie1@lsuhsc.edu

\section{INTRODUCTION}

The coronavirus disease (COVID-19) pandemic of 2020, caused by severe acute respiratory syndrome coronavirus 2 (SARS-CoV-2, initially referred to as 2019-nCoV), has resulted in challenges to essentially all sectors of global society ${ }^{1}$ and particularly to the health care sector. $^{2}$ The radiology community responded with a plethora of publications during the relatively short interval of rapid case increases at the beginning of 2020 . Experts across the globe, including the Radiological Society of North America (RSNA) and the American College of Radiology, ${ }^{3,4}$ provided recommendations on the utility of imaging in diagnosis and management of COVID-19.
Most of these recommendations, however, focus on computed tomography (CT) manifestations and not on chest radiographs (CXRs), with the prevailing theme that $\mathrm{CT}$ should be used sparingly, with a predilection for patients in the inpatient setting and not as a screening tool. ${ }^{3-5}$ Further, much attention was given to the temporal progression of disease as seen on $\mathrm{CT}^{6-14}$ specifically the increase in multiplicity and density of airspace opacities, ultimately coalescing into diffuse pulmonary opacification and directly correlating with worsening clinical symptoms. ${ }^{7}$ Disease progression from a CXR perspective was addressed relatively less. ${ }^{15-18}$ Some authors underscore their observations with respect to patient age, as incidence and severity of clinical outcomes appear to be greater in older individuals infected with 
SARS-CoV-2. ${ }^{19,20}$ In fact, 2020 publications from Li et al and Chen et al suggest that the majority of patients they studied with COVID-19 were older than 50 years and that disease progression appears to become more rapid with advances in age. ${ }^{7,21}$

This greater focus on CT is not surprising given the lower sensitivity of CXR vs CT for pulmonary disease in general. However, some centers have used CXR as an adjunct to clinical diagnosis in the early stages of COVID-19, given challenges such as prolonged turnaround time and test variability for reverse transcription polymerase chain reaction (RT-PCR) tests and infection issues related to CT scanning. ${ }^{22-25}$ Wong et al reported the sensitivity of CXR in detecting COVID-19 to be $69 \%$ compared to $91 \%$ for RT-PCR, ${ }^{16}$ but in the Wong et al study, radiologic evaluation was not geared toward the typical patterns of reported imaging manifestations of COVID-19 described in recent (2020) literature ${ }^{17,18}$ and in consensus statements from the RSNA. ${ }^{3}$ Instead, the study focused on the assessment of pulmonary edema and used a radiographic assessment of lung edema score. ${ }^{26}$

In this article, we address the utility of using a strict pattern of findings on CXR for the diagnosis of COVID-19 during early onset of symptoms in patients older than and younger than 50 years of age.

\section{METHODS}

Our institutional review board approved this retrospective study with waiver of Health Insurance Portability and Accountability Act authorization in accordance with federal regulations at 45 CFR $\$ 164.512(i)(2)(i i)$. Using the keyword "COVID," we queried the picture archiving and communication system for patient CXRs performed from January 1, 2020 to April 8, 2020. We then searched the medical record numbers associated with the CXRs in the electronic medical record system at University Medical Center in New Orleans, Louisiana, for patients who had undergone COVID-19 RT-PCR assay. Only patients who tested positive by RT-PCR during the same admission were included in the study. Patients with CXRs performed after 7 days of symptom onset were excluded so that we could narrow our assessment of CXR findings to the earliest stages of disease. Symptoms reported by patients at the time of presentation included fever, chills, night sweats, cough, shortness of breath, malaise, loss of appetite, and gastrointestinal complaints.

The query returned $275 \mathrm{CXRs}$, and 197 patients were confirmed to be RT-PCR positive. From the 197 patients who were RT-PCR-positive, $30 \mathrm{CXRs}$ from patients $>50$ years and 30 CXRs from patients $\leq 50$ years were chosen. All CXRs were confirmed to have been obtained within 7 days from onset of symptoms. Sex, age, and date of symptom onset were collected for the cohort.

\section{Image Acquisition and Analysis}

All CXRs were acquired as digital radiographs following usual institutional protocols using a Philips DigitalDiagnost system (Koninklijke Philips N.V.). The CXRs were acquired in anteroposterior projection with patients in upright $(22 / 60$, $37 \%)$, semi-upright $(29 / 60,48 \%)$, or supine $(9 / 60,15 \%)$ position, depending on the patients' dispositions at the time of image acquisition.

\section{Data Interpretation}

Two board-certified radiologists (with more than 5 and 10 years of experience interpreting CXRs following completion of residency) were blinded to RT-PCR results and clinical history and retrospectively reviewed $60 \mathrm{CXRs}$ in 60 patients obtained on average within 3 days of symptom onset. The two reviewers independently assigned one of the following patterns to each CXR: characteristic, atypical, or negative (Table 1). Discrepancies in the independent interpretations were resolved in a consensus session.

The characteristic COVID-19 pattern was defined in accordance with the most commonly reported chest imaging findings of COVID-19 in recent (2019 and 2020) literature, including the presence of bilateral patchy or confluent, bandlike ground glass opacity or consolidation in a peripheral and mid-to-lower lung zone distribution (Figures 1, 2, and 3). ${ }^{16,27-29}$ If the CXR showed some but not all these abnormalities, it was assigned to the atypical category. CXRs in the atypical category were subdivided into categories based on imaging findings being more suspicious than not for COVID19 or less suspicious for COVID-19. The atypical-more suspicious CXRs had a characteristic pattern seen only in one lung (Figures 4 and 5). The atypical-less suspicious CXRs were defined as abnormal but not necessarily showing a typical finding for COVID-19 pneumonia (Figures 6 and 7). A normal-appearing CXR was categorized as negative.

\section{Statistical Analysis}

Statistical analysis was performed using Excel 2018 (Microsoft Corporation). CXR characterizations-negative, atypical-more suspicious, atypical-less suspicious, and characteristic-were compared to patient age with a thresh-

Table 1. Chest Radiograph Imaging Classifications ${ }^{16,27-29}$

\begin{tabular}{ll}
\hline Classification & Description \\
\hline Characteristic COVID-19 appearance & $\begin{array}{r}\text { Presence of commonly reported chest imaging findings associated with COVID-19: } \\
\text { bilateral patchy or confluent band-like ground glass opacity or consolidation in a } \\
\text { peripheral and mid-to-lower lung zone distribution }\end{array}$ \\
$\begin{array}{l}\text { Atypical-more suspicious than not for } \\
\text { COVID-19 }\end{array}$ & $\begin{array}{r}\text { Findings suggestive of a diagnosis other than COVID-19 infection (eg, pulmonary } \\
\text { edema, atelectasis, interstitial changes) }\end{array}$ \\
$\begin{array}{l}\text { Atypical-less suspicious for COVID-19 } \\
\text { Negative }\end{array}$ & No abnormal findings \\
\hline
\end{tabular}




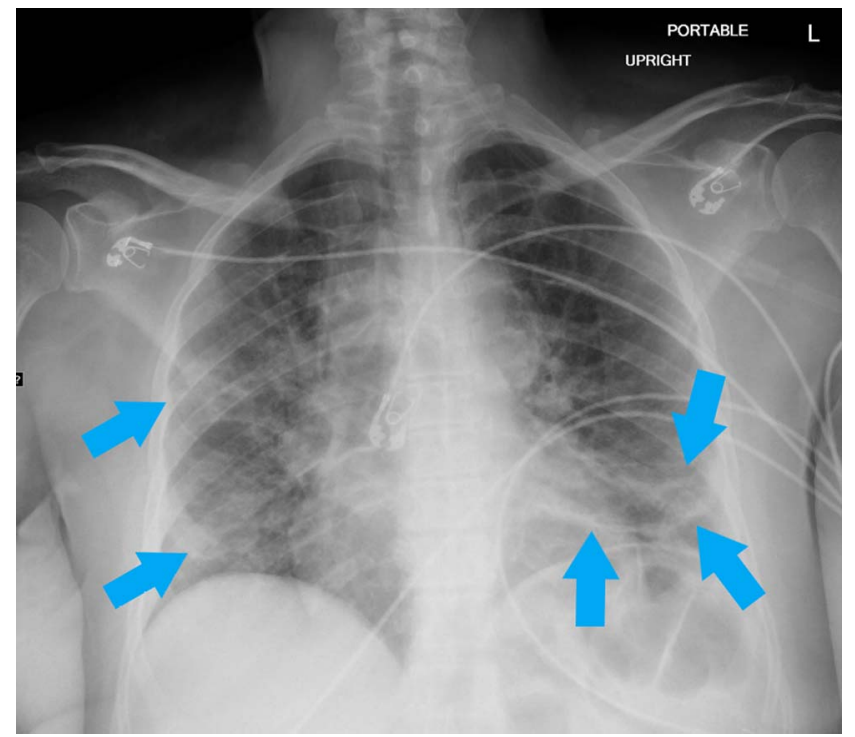

Figure 1. Portable upright anteroposterior radiograph of a 65-year-old female shows characteristic bilateral confluent, bandlike (arrows) consolidative opacity in a peripheral, mid-to-lower lung zone distribution admixed with patchy airspace opacity.

old of 50 years using a chi-square test of independence and Fisher exact test. Significance level was defined as $P<0.05$.

Interreader agreement was assessed using Cohen kappa

(к) coefficient. The 2 readers had substantial agreement on the evaluation of CXRs for COVID-19 $(\kappa=0.743)$, agreeing on 50 of the 60 (83\%) CXRs. ${ }^{30}$

\section{RESULTS}

Of the 60 patients included in the study, 30 (10 males, 20 females) were in the $>50$ years group and 30 (14 males, 16 females) were in the $\leq 50$ years group (Table 2$)$. The range

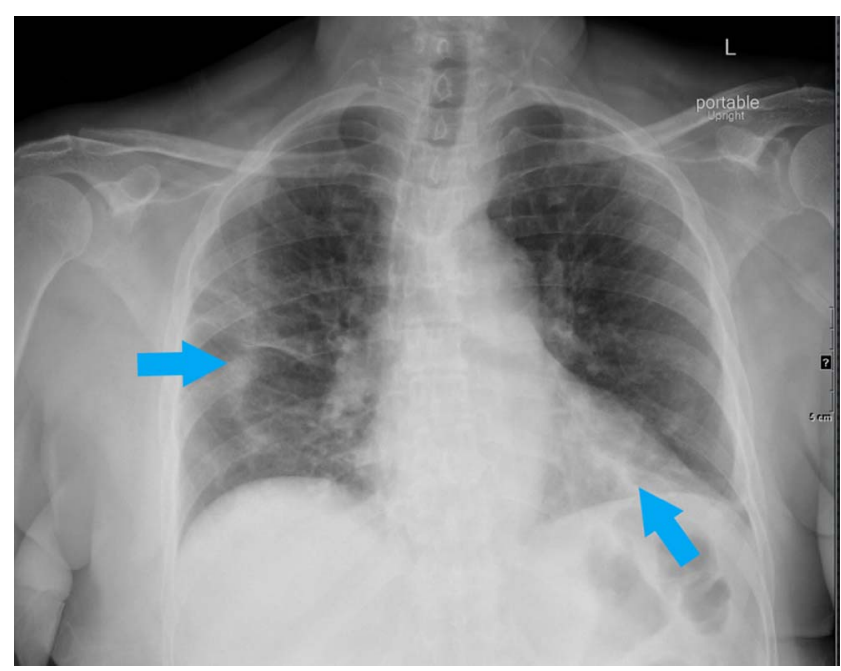

Figure 2. Portable upright anteroposterior radiograph of a 72-year-old female shows characteristic bilateral confluent, bandlike (arrows) consolidative opacity in periphery of the right mid- and left-lower lung zones.

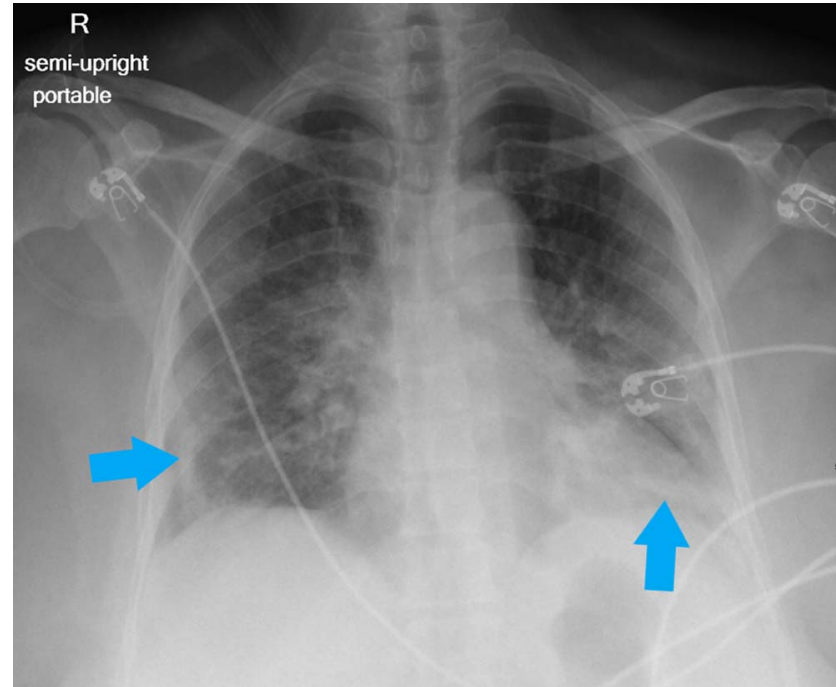

Figure 3. Portable semi-upright anteroposterior radiograph of a 66-year-old female shows characteristic bilateral confluent, bandlike (arrows) consolidative opacity in a peripheral, mid-to-lower lung zone distribution admixed with patchy airspace opacity, greatest at the left lung base.

of ages in the $>50$ years group was 52 to 88 years (mean, 65 years). The range in the younger group was 19 to 48 years (mean, 37 years). All 60 patients had a positive COVID-19 RT-PCR assay. Both the older and younger cohorts received CXRs an average of 3 days after the reported onset of symptoms, with a range of 0 to 5 days in the older group and a range of 1 to 7 days in the younger group.

\section{Imaging Findings}

Table 2 shows the imaging findings by classification and by group. Overall, $73 \%(44 / 60)$ of the patients included in

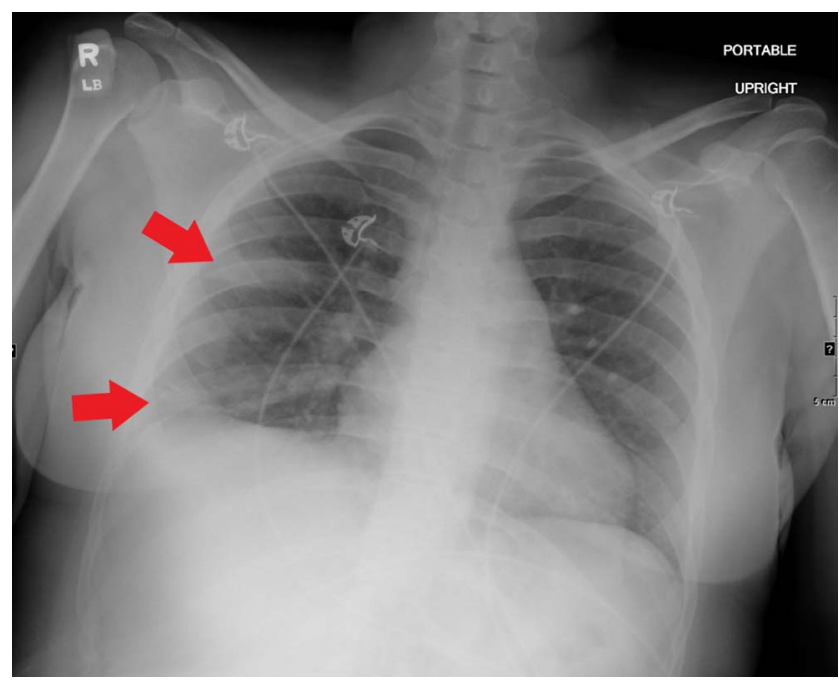

Figure 4. Portable upright anteroposterior radiograph of a 30-year-old female shows unilateral confluent, bandlike (arrows) consolidative opacity in the periphery of the right mid and lower lung zones. This pattern was considered atypical, but more suspicious for coronavirus disease 2019. 
Table 2. Imaging Results by Age Group and by Sex by Age Group

\begin{tabular}{|c|c|c|c|c|}
\hline \multirow[b]{2}{*}{ Variable } & \multicolumn{4}{|c|}{ Chest Radiograph Findings $\mathrm{s}^{\mathrm{a}}$} \\
\hline & Negative & $\begin{array}{c}\text { Characteristic } \\
\text { COVID-19 Appearance }\end{array}$ & $\begin{array}{l}\text { Atypical-More } \\
\text { Suspicious }\end{array}$ & $\begin{array}{l}\text { Atypical-Less } \\
\text { Suspicious }\end{array}$ \\
\hline \multicolumn{5}{|l|}{ Age, years } \\
\hline$>50(n=30)$ & $2(3)$ & $11(18)$ & $13(22)$ & $4(7)$ \\
\hline$\leq 50(n=30)$ & $14(23)$ & $4(7)$ & $5(8)$ & $7(12)$ \\
\hline \multicolumn{5}{|l|}{ Sex by age group, years } \\
\hline Male $>50(n=10)$ & $1(2)$ & $3(5)$ & $5(8)$ & $1(2)$ \\
\hline Female $>50(n=20)$ & $1(2)$ & $8(13)$ & $8(13)$ & $3(5)$ \\
\hline Male $\leq 50(n=14)$ & $8(13)$ & $3(5)$ & $0(0)$ & $3(5)$ \\
\hline Female $\leq 50(n=16)$ & $6(10)$ & $1(2)$ & $5(8)$ & $4(7)$ \\
\hline
\end{tabular}

Note: All percentages are calculated based on the total number of patients included in the study $(n=60)$.

${ }^{a}$ Classifications of chest radiograph findings are defined in Table 1.

this study had an abnormal CXR (Table 3 ). In the $>50$ years group, 93\% (28/30) had an abnormal CXR that was classified either as characteristic (classic COVID-19 findings) or as atypical. Seven percent $(2 / 30)$ of patients in the $>50$ years group had a negative CXR within the first week of presentation from symptom onset. In the same period from symptom onset, 53\% (16/30) of patients in the younger group had an abnormal CXR, and the other 47\% (14/30) had a normal CXR (Table 3 and Figure 8).

The relationship between age and CXR findings was statistically significant according to chi-square test ([2, $\mathrm{n}=60]=15.70 ; P=0.00039$ ). In addition, a Fisher exact test comparing negative and characteristic COVID-19 CXR find-

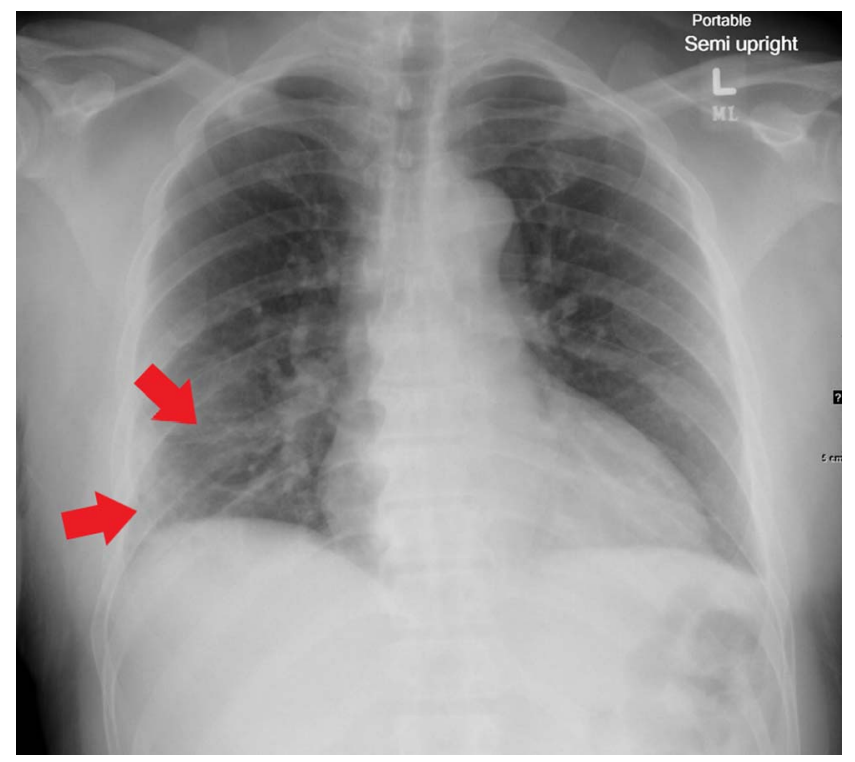

Figure 5. Portable semi-upright anteroposterior radiograph of a 56-year-old male shows unilateral thin bandlike (arrows) consolidative opacity in the periphery of the right mid and lower lung zones admixed with patchy airspace opacity. This pattern was considered atypical, but more suspicious for coronavirus disease 2019. ings to patient age determined that the relationship was statistically significant $(P=0.001)$. Patients $>50$ years were more likely to have characteristic and abnormal suspicious CXR findings during the first week after symptom onset.

\section{DISCUSSION}

Our finding of $73 \%$ CXR abnormalities within the first week of symptom onset in the combined cohort closely approximates other reported measures of sensitivity, such as Wong et al at $69 \%{ }^{16}$ and Hosseiny et al in which $75 \%$ of patients had a similar distribution of findings in early imaging. ${ }^{31}$ When patient age is considered, we demonstrated a 93\% sensitivity in patients $>50$ years and $53 \%$ in patients $\leq 50$ years in the first 7 days after symptom onset.

Older age has been associated with poorer outcomes in COVID-19. ${ }^{31,32}$ In the United States, $80 \%$ of deaths have

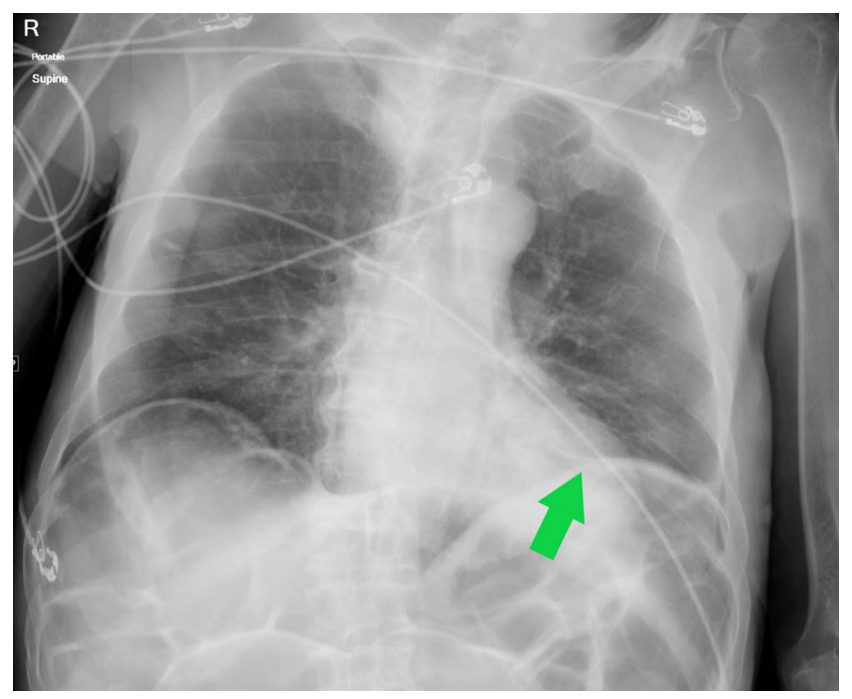

Figure 6. Portable supine anteroposterior radiograph of an 80-year-old male shows unilateral discoid (arrow) opacity along the left hemidiaphragm, typical of subsegmental atelectasis. This pattern was considered atypical and less suspicious for coronavirus disease 2019. 


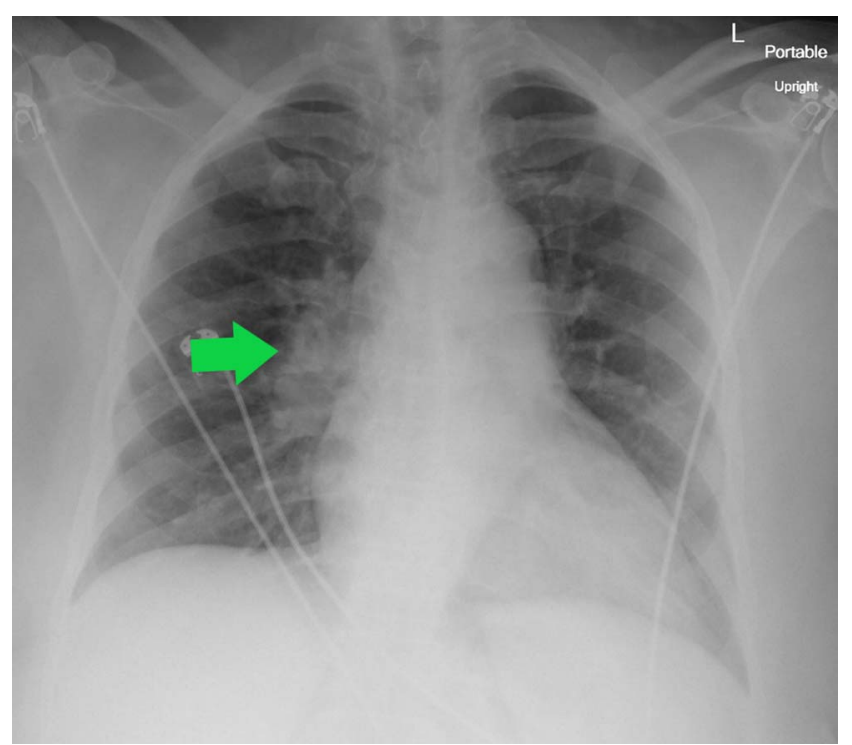

Figure 7. Portable upright anteroposterior radiograph of a 68-year-old female shows prominence of the right hilar shadow (arrow) and otherwise clear lungs. This pattern was considered atypical and less suspicious for COVID-19.

occurred in patients $\geq 65$ years, and patients $\geq 85$ years have an overwhelming percentage of severe outcomes, mirroring the reported initial experience in China. ${ }^{33}$

Many associated factors have been addressed in the literature, such as angiotensin-converting enzyme 2 (ACE2) receptor, identified as a target of SARS-CoV-2. ${ }^{34}$ One suggestion is that different levels of ACE2 in younger and older individuals, in particular with respect to decreasing levels within the aging tissues of the lungs and heart, may have an effect on the severity of COVID-19-related disease. ${ }^{35}$ Liu et al reported high levels of angiotensin in correlation with increased viral load of SARS-CoV-2. ${ }^{36}$ In addition, noncommunicable illnesses associated with advancing age, such as hypertension and heart disease, in combination with their associated therapies are generally thought to hinder immune response. ${ }^{37}$ Other age-related links in infectious disease have also been noted. For example, Rivers et al reported that the major risk factors for progression of disease in Middle East respiratory syndrome (MERS-CoV) were underlying comorbidities, such as cardiac disease and diabetes, and age, and the reported mean age in patients considered most severely ill was 57 years. ${ }^{38}$

Table 3. Overall Imaging Results by Age Group

\begin{tabular}{lcc}
\hline & \multicolumn{2}{c}{ Chest Radiograph Findings ${ }^{\mathbf{a}}$} \\
\cline { 2 - 3 } Age Group, years & Normal & Abnormal \\
\hline$>50(n=30)$ & $2(7)$ & $28(93)$ \\
$\leq 50(n=30)$ & $14(47)$ & $16(53)$ \\
\hline
\end{tabular}

Note: Percentages are calculated by row $(n=30)$.

${ }^{a} A l l$ negative radiographs are classified as normal. The abnormal category includes radiographs classified as characteristic, atypical-more suspicious, and atypical-less suspicious.

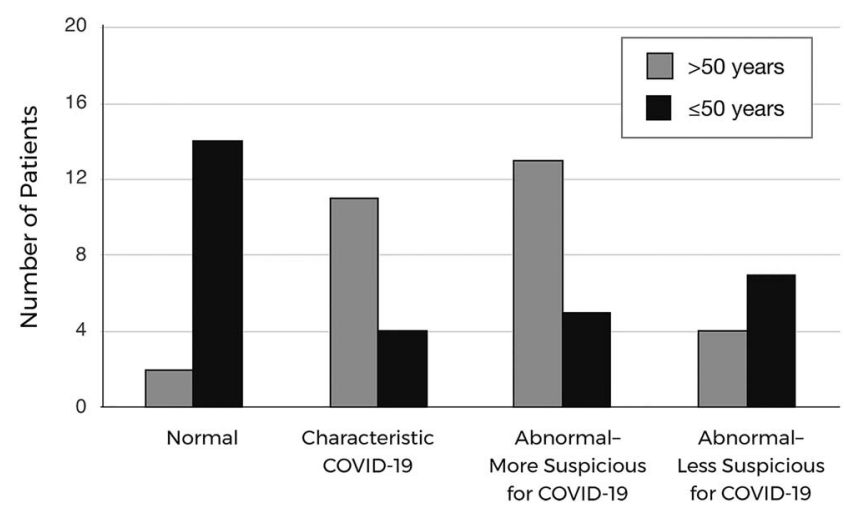

Figure 8. Chest radiograph findings for patients in the $\mathbf{2}$ age cohorts.

While a new strain of coronaviridae, SARS-CoV-2, has been identified as the causative pathogen for the COVID19 pandemic, the outbreak of severe pulmonary disease caused by a strain of this viral family is not unique. MERSCoV and severe acute respiratory syndrome (SARS-CoV) are the most salient examples. While no outbreaks of SARSCoV have been reported since April 2004, MERS-CoV cases have been reported as recently as January $2020 .{ }^{39}$ Both syndromes have imaging features similar to COVID-19, ${ }^{31}$ which is important because symptoms associated with COVID-19 have been reported to be milder than those of MERS-CoV, ${ }^{40}$ allowing for a potentially greater role for imaging in disease management, particularly in patients at greater risk for disease severity - the older population.

Much is still unknown regarding patients' future immunity to COVID-19 after convalescence. The immune system of older adults undergoes numerous age-related changes, collectively termed immune senescence, that leave older adults particularly vulnerable to new, emerging infectious diseases, such as possible reinfection by COVID-19 or infection with new variants. ${ }^{33}$

Limitations of our study include the small sample size at a single institution, lack of follow-up serial CXR or subsequent CT scans that may have been performed, and inclusion of only RT-PCR-positive patients. Selection bias was also present because all patients were chosen from a subset that received imaging, potentially because of a more severe course of illness. Additionally, descriptions of the temporal progression of disease used in this study have been defined almost entirely by recent publications on CT findings. With the rapid increase in number of abnormalities and distribution peaking 6 to 11 days after onset, identifying how patients with a worsening course presented on early CXR will be important. ${ }^{14}$ Despite global vaccination efforts, there is still concern that the disease could produce enough severe illness to further strain some health care infrastructures, especially with new variants circulating in the community. As a result, health care providers need to be able to predict which patients will have a more complex course of illness. ${ }^{41}$ Early CXR correlation with patient course of illness and outcomes could be critical in anticipating what resources will be needed in the coordination of care for COVID-19 patients. 


\section{CONCLUSION}

We conclude that CXR in patients $>50$ years within 1 week of symptom onset in COVID-19 offers potential benefits, whereas a negative CXR in patients $\leq 50$ years has limited sensitivity. Imaging obtained early in presentation of disease, especially for patients older than 50 years, may help to inform an estimate of resource use.

\section{ACKNOWLEDGMENTS}

We are grateful to the following individuals for their assistance, guidance, and helpful discussions: Robert Karl, MD, John-Paul Grenier, MD, Bridgette Workman, Jadawn Darensbourg, and Wanda Sandoval. The authors have no financial or proprietary interest in the subject matter of this article.

\section{REFERENCES}

1. Nicola M, Alsafi Z, Sohrabi C, et al. The socio-economic implications of the coronavirus pandemic (COVID-19): a review. Int J Surg. 2020;78:185-193. doi: 10.1016/j.ijsu.2020.04.018

2. Tanne JH, Hayasaki E, Zastrow M, Pulla P, Smith P, Rada AG. Covid-19: how doctors and healthcare systems are tackling coronavirus worldwide. BMJ. 2020;368:m1090. doi: 10.1136/bmj.m1090

3. Simpson S, Kay FU, Abbara S, et al. Radiological Society of North America Expert consensus statement on reporting chest CT findings related to COVID-19. Endorsed by the Society of Thoracic Radiology, the American College of Radiology, and RSNA. Radiol Cardiothorac Imaging. 2020;2(2). doi: $10.1148 /$ ryct.2020200152

4. ACR recommendations for the use of chest radiography and computed tomography (CT) for suspected COVID-19 infection. American College of Radiology. March 11, 2020. Updated March 22, 2020. Accessed March 8, 2021. www.acr.org/Advocacy-and-Economics/ACR-PositionStatements/Recommendations-for-Chest-Radiography-andCT-for-Suspected-COVID19-Infection

5. Rubin GD, Ryerson CJ, Haramati LB, et al. The role of chest imaging in patient management during the covid-19 pandemic: a multinational consensus statement from the Fleischner Society. Chest. 2020;158(1):106-116. doi: 10.1016/j.chest.2020.04.003

6. Ai T, Yang Z, Hou H, et al. Correlation of chest CT and RT-PCR testing in coronavirus disease 2019 (COVID-19) in China: a report of 1014 cases. Radiology. 2020;296(2):E32-E40. doi: 10.1148/radiol.2020200642

7. Li M, Lei P, Zeng B, et al. Coronavirus disease (COVID-19): spectrum of $\mathrm{CT}$ findings and temporal progression of the disease. Acad Radiol. 2020;27(5):603-608. doi: 10.1016/j.acra.2020.03.003

8. Zhou S, Wang Y, Zhu T, Xia L. CT features of coronavirus disease 2019 (COVID-19) pneumonia in 62 patients in Wuhan, China. AJR Am J Roentgenol. 2020;214(6):1287-1294. doi: 10.2214/ajr.20.22975

9. Salehi S, Abedi A, Balakrishnan S, Gholamrezanezhad A. Coronavirus disease 2019 (COVID-19): a systematic review of imaging findings in 919 patients. AJR Am J Roentgenol. 2020;215(1):87-93. doi: 10.2214/AJR.20.23034

10. Cheng Z, Lu Y, Cao Q, et al. Clinical features and chest CT manifestations of coronavirus disease 2019 (COVID-19) in a single-center study in Shanghai, China. AJR Am J Roentgenol. 2020;215(1):121-126. doi: 10.2214/ajr.20.22959
11. Guan CS, Lv ZB, Yan S, et al. Imaging features of coronavirus disease 2019 (COVID-19): evaluation on thin-section CT. Acad Radiol. 2020;27(5):609-613. doi: 10.1016/j.acra.2020.03.002

12. Pan $F, Y e T$, Sun $P$, et al. Time course of lung changes on chest CT during recovery from 2019 novel coronavirus (COVID-19). Radiology. 2020;295(3):715-721. doi: 10.1148/radiol.2020200370

13. Bernheim $A$, Mei $X$, Huang $M$, et al. Chest $C T$ findings in coronavirus disease-19 (COVID-19): relationship to duration of infection. Radiology. 2020;295(3):200463. doi: $10.1148 /$ radiol.2020200463

14. Wang Y, Dong C, Hu Y, et al. Temporal changes of CT findings in 90 patients with COVID-19 pneumonia: a longitudinal study. Radiology. 2020;296(2):E55-E64. doi: 10.1148/radiol.2020200843

15. Jacobi A, Chung M, Bernheim A, Eber C. Portable chest x-ray in coronavirus disease-19 (COVID-19): a pictorial review. Clin Imaging. 2020;64:35-42. doi: 10.1016/j.clinimag.2020.04.001

16. Wong HYF, Lam HYS, Fong AHT, et al. Frequency and distribution of chest radiographic findings in COVID-19 positive patients. Radiology. 2020;296(2):E72-E78. doi: 10.1148/radiol.2020201160

17. Ng MY, Lee EYP, Yang J, et al. Imaging profile of the COVID-19 infection: radiologic findings and literature review. Radiol Cardiothorac Imaging. 2020;2(1). doi: 10.1148/ryct.2020200034

18. Yoon SH, Lee KH, Kim JY, et al. Chest radiographic and CT findings of the 2019 novel coronavirus disease (COVID-19): analysis of nine patients treated in Korea. Korean J Radiol. 2020;21(4):494-500. doi: 10.3348/kjr.2020.0132

19. Garnier-Crussard A, Forestier E, Gilbert T, Krolak-Salmon P. Novel coronavirus (COVID-19) epidemic: what are the risks for older patients? J Am Geriatr Soc. 2020;68(5):939-940. doi: $10.1111 /$ jgs.16407

20. Sun P, Lu X, Xu C, Sun W, Pan B. Understanding of COVID-19 based on current evidence. J Med Virol. 2020;92(6):548-551. doi: $10.1002 / j m v .25722$

21. Chen J, Qi T, Liu L, et al. Clinical progression of patients with COVID-19 in Shanghai, China. J Infect. 2020;80(5):e1-e6. doi: 10.1016/j.jinf.2020.03.004

22. Asperges E, Novati S, Muzzi A, et al. Rapid response to COVID-19 outbreak in northern Italy: how to convert a classic infectious disease ward into a COVID-19 response centre. $J$ Hosp Infect. 2020;105(3):477-479. doi: 10.1016/j.jhin.2020.03.020

23. Chen TY, Lai HW, Hou IL, et al. Buffer areas in emergency department to handle potential COVID-19 community infection in Taiwan. Travel Med Infect Dis. 2020;36:101635. doi: 10.1016/j.tmaid.2020.101635

24. Hare SS, Rodrigues J, Nair A, Robinson G. Lessons from the frontline of the covid-19 outbreak. BMJ Opinion. March 20, 2020. Accessed March 22, 2020. blogs.bmj.com/bmj/2020/ 03/20/lessons-from-the-frontline-of-the-covid-19-outbreak/

25. Imaging the coronavirus disease COVID-19. healthcare-in-europe.com. March 16, 2020. Accessed March 23, 2020. healthcare-in-europe.com/en/news/imaging-thecoronavirus-disease-covid-19.html

26. Warren MA, Zhao Z, Koyama T, et al. Severity scoring of lung oedema on the chest radiograph is associated with clinical outcomes in ARDS. Thorax. 2018;73(9):840-846. doi: 10.1136/thoraxjnl-2017-211280

27. Kong W, Agarwal PP. Chest imaging appearance of COVID-19 infection. Radiol Cardiothorac Imaging. 2020;2(1). doi: $10.1148 /$ ryct.2020200028 
28. Bai HX, Hsieh B, Xiong Z, et al. Performance of radiologists in differentiating COVID-19 from non-COVID-19 viral pneumonia at chest CT. Radiology. 2020;296(2):E46-E54. doi: 10.1148/radiol.2020200823

29. Smith DL, Grenier JP, Batte C, Spieler B. A characteristic chest radiographic pattern in the setting of COVID-19 pandemic. Radiol Cardiothorac Imaging. 2020;2(5). doi: $10.1148 /$ ryct.2020200280

30. Landis JR, Koch GG. The measurement of observer agreement for categorical data. Biometrics. 1977;33(1):159-174.

31. Hosseiny M, Kooraki S, Gholamrezanezhad A, Reddy S, Myers L. Radiology perspective of coronavirus disease 2019 (COVID-19): lessons from severe acute respiratory syndrome and Middle East respiratory syndrome. AJR Am J Roentgenol. 2020;214(5):1078-1082. doi: 10.2214/AJR.20.22969

32. People at increased risk. Centers for Disease Control and Prevention. Accessed April 15, 2020. www.cdc.gov/coronavirus/ 2019-ncov/need-extra-precautions/people-at-higher-risk.html

33. Nikolich-Zugich J, Knox KS, Rios CT, Natt B, Bhattacharya D, Fain MJ. SARS-CoV-2 and COVID-19 in older adults: what we may expect regarding pathogenesis, immune responses, and outcomes. Geroscience. 2020;42(2):505-514. doi: 10.1007/s11357-020-00186-0

34. Brojakowska A, Narula J, Shimony R, Bander J. Clinical implications of SARS-Cov2 interaction with renin angiotensin system: JACC review topic of the week. J Am Coll Cardiol. 2020;75(24):3085-3095. doi: 10.1016/j.jacc.2020.04.028
35. Vaduganathan M, Vardeny O, Michel T, McMurray JJV, Pfeffer MA, Solomon SD. Renin-angiotensin-aldosterone system inhibitors in patients with Covid-19. N Engl J Med. 2020;382(17):1653-1659. doi: 10.1056/NEJMsr2005760

36. Liu Y, Yang Y, Zhang C. Clinical and biochemical indexes from 2019-nCoV infected patients linked to viral loads and lung injury. Sci China Life Sci. 2020;63(3):364-374.

37. Liguori I, Russo G, Curcio F, et al. Oxidative stress, aging, and diseases. Clin Interv Aging. 2018;13:757-772. doi: $10.2147 / \mathrm{CIA} . S 158513$

38. Rivers CM, Majumder MS, Lofgren ET. Risks of death and severe disease in patients with Middle East respiratory syndrome coronavirus, 2012-2015. Am J Epidemiol. 2016;184(6):460-464. doi: 10.1093/aje/kww013

39. Middle East respiratory syndrome coronavirus (MERS-CoV) the Kingdom of Saudi Arabia. World Health Organization. Updated February 24, 2020. Accessed February 24, 2020. www. who.int/csr/don/24-february-2020-mers-saudi-arabia/en/

40. Kannan S, Shaik Syed Ali P, Sheeza A, Hemalatha K. COVID-19 (novel coronavirus 2019) - recent trends. Eur Rev Med Pharmacol Sci. 2020;24(4):2006-2011. doi: 10.26355/eurrev_202002_20378

41. Emanuel EJ, Persad G, Upshur R, et al. Fair allocation of scarce medical resources in the time of Covid-19. N Engl J Med. 2020;382(21):2049-2055. doi: 10.1056/NEJMsb2005114

This article meets the Accreditation Council for Graduate Medical Education and the American Board of Medical Specialties Maintenance of Certification competencies for Patient Care, Medical Knowledge, and Practice-Based Learning and Improvement.

(C2021 by the author(s); licensee Ochsner Journal, Ochsner Clinic Foundation, New Orleans, LA. This article is an open (c) (i) access article distributed under the terms and conditions of the Creative Commons Attribution (CC BY) license (creativecommons.org/licenses/by/4.0/legalcode) that permits unrestricted use, distribution, and reproduction in any medium, provided the original author(s) and source are credited. 